\title{
STABLE MARRIAGE PROBLEM WITH TIES AND INCOMPLETE BOUNDED LENGTH PREFERENCE LIST UNDER SOCIAL STABILITY
}

\author{
Ashish Shrivastava and C. Pandu Rangan \\ Department of Computer Science and Engineering, \\ Indian Institute of Technology, Madras, Chennai, India \\ ashish3586@gmail.com, prangan55@gmail.com
}

\begin{abstract}
We consider a variant of socially stable marriage problem where preference lists may be incomplete, may contain ties and may have bounded length. In real world application like NRMP and Scottish medical matching scheme such restrictions arise very frequently where set of agents (man/woman) is very large and providing a complete and strict order preference list is practically in-feasible. In presence of ties in preference lists, the most common solution is weakly socially stable matching. It is a fact that in an instance, weakly stable matching can have different sizes. This motivates the problem of finding a maximum cardinality weakly socially stable matching.

In this paper, we find maximum size weakly socially stable matching for an instance of Stable Marriage problem with Ties and Incomplete bounded length preference list with Social Stability. The motivation to consider this instance is the known fact, any larger instance of this problem is NP-hard.
\end{abstract}

\section{KEYWORDS}

Stable Marriage Problem, Socially Stable Matching, Bipartite Matching, Stable Marriage Problem with Ties and Incomplete list.

\section{INTRODUCTION}

The Stable marriage problem was first introduced by Gale and Shapley in 1962 [1]. The classical instance $I$ of the stable marriage problem has a set of $n$ men $U$, a set of $n$ women $W$ and preference lists of men over women and vice versa. Each preference list contains all members of opposite sex in a strict order. A man $m_{i}$ and a woman $w_{j}$ are called acceptable to each other in I nstance $I$ if $m_{i}$ is in preference list of $w_{j}$ and $w_{j}$ is in preference list of $m_{i}$. Let $\alpha$ is the set of all acceptable pairs in the instance $I$. A matching $M$ is a set of independent pairs $\left(m_{i}, w_{j}\right)$ such that $m_{i}$ $\in U$ and $w_{j} \in W$. If $\left(m_{i}, w_{j}\right) \in M$, we say that $\mathrm{m}_{\mathrm{i}}$ is matched to $w_{j}$ in $M$ and vice versa and we denote $M\left(m_{i}\right)=w_{j}$ and $M\left(w_{j}\right)=m_{i}$.

Jan Zizka et al. (Eds) : CCSIT, SIPP, AISC, CMCA, SEAS, CSITEC, DaKM, PDCTA, NeCoM - 2016 pp. 53-62, 2016. ( ) CS \& IT-CSCP 2016

DOI : $10.5121 /$ csit.2016.60106 
A pair $\left(m_{i}, w_{j}\right) \notin \mathrm{M}$ is called a blocking pair for matching $M$ if both $m_{i}$ and $w_{j}$ prefer each other to their partners in $M$. A matching $M$ is called a stable matching iff there is no blocking pair with respect to $M$. Gale and Shapley gave a deferred acceptance algorithm and proved that every instance $I$ of the stable marriage problem admits a stable matching which can be found in polynomial time [1].

The largest and one of the best known applications of Hospitals Residents problem is National Resident Matching Program (NRMP) and Scottish medical matching scheme which match graduated medical students (residents) with their preferred hospitals on the basis of both side preference lists.

The research work in the field of The Stable Marriage Problem has a long history. As we have mentioned earlier, the first problem on stable marriage problem was introduced by Gale and Shapley in 1962. After that lots of variation on first problem came into the picture. Some major variations are Stable Marriage problem with Ties (SMT), Stable Marriage problem with Incomplete list (SMI), Stable Marriage problem with Ties and Incomplete list (SMTI) and Stable Marriage problem with Bounded length preference lists.

\subsection{Stable marriage problem with ties (SMT)}

In Stable Marriage problem with Ties, each man can give a preference list over a set of women, where two or more women can hold the same place (ties) in the preference list and vice-versa. In SMT there are three notion of stability: weak stability, strong stability and super stability [2,3]. A blocking pair $\left(m_{i}, w_{j}\right) \notin M$ with respect to a weakly stable matching $M$ can be defined as follows: (a) $m_{i}$ and $w_{j}$ are acceptable to each other. (b) $m_{i}$ strictly prefers $w_{j}$ to $M\left(m_{i}\right)$ (partner of $m_{i}$ in matching $M$ ) (c) $w_{j}$ strictly prefers $m_{i}$ to $M\left(w_{j}\right)$. For an instance $I$ of weakly stable matching problem, a weakly stable matching $M$ always exist and can be found in polynomial time [3].

A blocking pair $\left(m_{i}, w_{j}\right) \notin M$ with respect to a strongly stable matching $M$ can be defined as follows: (a) $m_{i}$ and $w_{j}$ are acceptable to each other. (b) $m_{i}$ strictly prefer $w_{j}$ to $M\left(m_{i}\right)$ and $w_{j}$ is indifferent between $m_{i}$ and $M\left(w_{j}\right)$ and vice-versa.

A blocking pair $\left(m_{i}, w_{j}\right) \notin M$ with respect to a super stable matching $M$ can be defined as follows: (a) $m_{i}$ and $w_{j}$ are acceptable to each other. (b) both $m_{i}$ and $w_{j}$ either strictly prefer each other to their partners $M$ or indifferent between them. There could be an instance $I$ that have neither super nor strongly stable matching but there is an algorithm which can find super and strong stable matching in $I$ (if exist) in polynomial time [4]. Among these three stability notions, weak stability has received most attention in the literature [5-12].

\subsection{Stable marriage problem with incomplete lists (SMI)}

Stable Marriage with Incomplete list (SMI) is another variation of stable marriage problem in which number of men and women in an instance $I$ need not be same. Each man and woman can give a preference list over a subset of opposite sex. For an instance $I$ a pair $\left(m_{i}, w_{j}\right)$ is called blocking pair with respect to a matching $M$ if: (a) $m_{i}$ and $w_{j}$ are acceptable to each other (b) $m_{i}$ is either unmatched in $M$ or prefer $w_{j}$ to $M\left(m_{i}\right)$ (c) $w_{j}$ is either unmatched in $M$ or prefer $m_{i}$ to $M\left(w_{j}\right)$. A matching $M$ is called stable if there is no blocking pair with respect to $M$.

In an instance $I$ of SMI we can partition the set of men and women such that, one partition have those men and women which have partners in all stable matching and other partition have those 
men and women which are unmatched in all stable matching [13].

\subsection{Stable marriage problem with ties and incomplete lists (SMTI)}

Stable Marriage with Ties and Incomplete list (SMTI) is an extension of classical stable marriage problem in which number of men and women in an instance $I$ need not be same. Each man gives a preference list over a subset of women and vice-versa. Each preference list may contain ties (two or more men/women have same rank). A pair $\left(m_{i}, w_{j}\right) \notin \mathrm{M}$ forms a blocking pair with respect to matching $M$ if (a) Both $m_{i}$ and $w_{j}$ are acceptable to each other and (b) $m_{i}$ is either unmatched or strictly prefers $w_{j}$ to $M\left(m_{i}\right)$ and (c) $w_{j}$ is either unmatched or strictly prefers $m_{i}$ to $M\left(w_{j}\right)$. A matching $M$ is called a weakly stable matching if there is no blocking pair with respect to $M$.

It is known that a weakly stable matching in an instance $I$ of SMTI can have different sizes and finding maximum cardinality weakly stable matching is an NP-hard problem [6]. NP-hardness holds even if only one tie of size 2 occurs on men's preference list at the tail and women's preference list contain no ties [6].

\subsection{Stable marriage problem with bounded length preference lists.}

The idea behind bounded length preference list is, in case of large scale matching problems, the preference list of at-least one side of agent tend to be short. An example of large scale matching is Scottish medical matching scheme [14] where each student is required to rank only three hospitals in their preference list. This variation leads to a question, whether problem of finding maximum size stable matching becomes simpler? (For an instance, with one side or both sided bounded preference list).

Suppose $(p, q)$-MAX SMTI denotes such variation on MAX SMTI problem (finding maximum size matching in an instance of SMTI) where each man can give at-most $p$ women in his preference list and each woman can give at-most $q$ men in her preference list. Halldorsson et al. [7] showed that $(4,7)$-MAX SMTI is NP-hard and not approximable within some $\delta>1$ unless $\mathrm{P}=$ NP. A reduction from Minimum Vertex Cover to MAX SMTI, shows that later problem cannot be approximable within 21/19 unless $\mathrm{P}=\mathrm{NP}$ [9]. Another study in [15] uses NP-hard restriction of minimum vertex cover of graph of minimum degree 3 in producing NP-hard result for $(5,5)$ MAX SMTI. Irving et al. [16] shows that $(3,4)$-MAX SMTI is NP-hard and not approximable within $\delta>1$ unless $\mathrm{P}=\mathrm{NP}$.

\section{RELATED WORK}

Another variation of stable marriage problem is socially stable marriage problem. An instance $I^{\prime \prime}$ of socially stable marriage problem can be defined by $(I, G)$ where $I$ is an instance of classical stable marriage problem and $G=(U \cup W, A)$ is a social network graph. Here $U$ and $W$ are set of men and women respectively and $A$ is set of man woman pair who knows each other in social network $G$. Set $A$ is called the set of acquainted pairs which is the subset of all acceptable pairs $(A \subseteq \alpha)$. A marriage $M$ is called socially stable marriage if there is no socially blocking pair with respect to $M$. A socially blocking pair $\left(m_{i}, w_{j}\right) \notin M$ is defined as follows: (a) both $m_{i}$ and $w_{j}$ prefers each other to their partner in $M$ and (b) $m_{i}$ and $w_{j}$ are connected in social network $G$.

In large scale matching like NRMP and Scottish medical matching scheme, social stability is a useful notion in which members of blocking pair block a matching $M$ only if they know the 
existence of each other. Thus the notion of social stability allows us to increase the cardinality of matching without taking care of those pairs which are not socially connected in social network graph.

The work in this paper is motivated by the work of Irving et al. [16] where they study about stable marriage problem with ties and bounded length preference list. They show that if each man's list is of length at most two and women's lists are of unbounded length with ties, we can find a maximum size weakly stable matching in polynomial time.

Our work in this paper is also motivated by the work of Askaladis et al. [17] where they study about socially stable matching problem with bounded length preference list. They gave a $O\left(n^{3 / 2}\right.$ $\log n)$ time algorithm for $(2, \infty)$ - MAX SMISS problem. Where $(2, \infty)$-MAX SMISS problem is to find a maximum size socially stable matching in an instance of stable marriage problem with incomplete list under social stability, where each man's list is of length at most two (without ties) and women's lists are of unbounded length (without ties).

\section{OUR CONTRIBUTION}

In an instance $I$ of $(2, \infty)$-MAX SMISS problem if we include ties on both side preference lists, where the length of a tie could be arbitrary, this instance converts into an instance $I^{\prime}$ of $(2, \infty)$ MAX SMTISS. In this paper we will show that we can find maximum size weakly socially stable matching in instance $I$ ' in polynomial time. Due to presence of ties in both side preference lists there are three notion of stability: weak, strong and super. In this paper we are considering maximum size weakly stable matching in $I^{\prime}$ of $(2, \infty)$-MAX SMTISS.

As we mention earlier, Irving et al. [16] shows that (3, 4)-MAX SMTI is NP-hard and not approximable within $\delta>1$ unless $\mathrm{P}=\mathrm{NP}$. It follows that the complexity status of $(3, \infty)$-MAX SMTI is also NP-hard. Similarly socially stable variation of $(3, \infty)$-MAX SMTI problem, "(3, $\infty)$-MAX Weakly SMTISS" is also NP-hard.

Given an instance I' of $(2, \infty)$-MAX Weakly SMTISS (Stable Marriage problem with Ties and Incomplete bounded length preference list under Social Stability), we present an algorithm that gives a maximum size weakly socially stable matching with time complexity $O\left(n^{3 / 2} \log n\right)$, where $n$ is the total number of men and women in the instance $I$.

\section{Stable Marriage Problem With TiES AND InCOMPlete BOUNDED LIST UNDER SOCIAL STABILITY (SMTISS)}

An instance of Stable Marriage Problem with Ties and Incomplete bounded list under Social Stability (SMTISS) can be defined by $(I, G)$ where I is the instance of SMTI and $G=(U \cup W, A)$, where $A$ (the set of all acceptable pairs). A man $m_{i}$ and a woman $w_{j}$ are called socially connected to each other in graph $G$ if $\left(m_{i}, w_{j}\right) \in A$. Each preference list is a partial order on a subset of opposite sex. A matching $M$ is called weakly socially stable if there is no socially blocking pair. A pair $\left(m_{i}, w_{j}\right) \notin M$ is a socially blocking pair if (a) $\left(m_{i}, w_{j}\right) \in A$ and (b) $m_{i}$ is either unmatched or strictly prefers $w_{j}$ to his partner in $M$ and (c) $w_{j}$ is either unmatched or strictly prefers $m_{i}$ to her partner in $M$. In general, for any instance $I$ of SMTISS problem, one of the aim is to compute a maximum cardinality socially stable matching (weakly, strong, super etc). In an incomplete tied preference list, arbitrary breaking of ties need not always lead to a maximum weakly socially stable matching. The following example shows that if we break ties arbitrarily we can find 
weakly socially stable matching of different sizes.

\begin{tabular}{|c|c|c|}
\hline Example: & Men's preference lists & Women's preference lists \\
\hline & $\mathrm{m}_{1}:\left(\underline{\mathrm{w}}_{1}, \mathrm{w}_{2}\right)$ & $\mathrm{w}_{1}: \underline{\mathrm{m}}_{1}, \mathrm{~m}_{2}$ \\
\hline & $\mathrm{m}_{2}: \mathrm{w}_{1}$ & $\mathrm{w}_{2}: \mathrm{m}_{1}$ \\
\hline
\end{tabular}

In above example the underline shows a social connection in $\mathrm{G}$. Here man $\mathrm{m}_{1}$ has a social connection with woman $\mathrm{w}_{1}$. Observe that if we break the tie of $m_{1}$ as $m_{1}: \underline{w}_{l}, w_{2}$ then maximum weakly socially stable matching will be $\left\{\left(m_{1}, w_{1}\right)\right\}$ of size 1 and if we break tie of $m_{1}$ as $m_{1}: w_{2}$, $\underline{w}_{1}$ then maximum weakly socially stable matching will be $\left\{\left(\mathrm{m}_{1}, \mathrm{w}_{2}\right),\left(m_{2}, w_{1}\right)\right\}$ of size 2 . The above example motivates us to find maximum cardinality weakly socially stable matching in an instance $I$ of SMTISS.

Observe that if we restrict the length of all ties equal to 1 in an instance $I$ of SMTISS then it will reduce into an instance $I^{\prime \prime}$ of SMISS. Since it is known that finding a maximum cardinality socially stable matching in an instance of SMISS is NP-complete [17], finding a maximum cardinality weakly socially stable matching in an instance of SMTISS is also NP-complete. Askalidis et al. showed that the problem $(2, \infty)$-MAX SMISS $((2, \infty)$-MAX SMTISS with ties length 1) is solvable in polynomial time [17], this result directed us to a more general version called $(2, \infty)$-MAX Weakly SMTISS problem where ties length could be two or more. It may seem that one can consider that if we break the ties arbitrary and apply $(2, \infty)$-Max SMISS algorithm then we can find maximum cardinality weakly socially stable matching for $(2, \infty)$ SMTISS instance, but this is not always true. We can verify this by above example.

\subsection{ALGORITHM FOR (2, $\infty)$-MAX WEAKLY SMTISS}

The objective of this problem is to find a maximum cardinality weakly socially stable matching in SMTI instance under social stability, where each man can give a preference list of length at most two and each woman can give unbounded length incomplete list, with or without ties of any length. We present an $O\left(n^{3 / 2} \log n\right)$ time algorithm for this problem. Similar to $(2, \infty)$-MAX SMISS given in [17], this algorithm also completes in three phases. In phase 1 we delete all pairs which can never belong to any weakly socially stable matching. The intuition behind phase 1 is, if there is a man $m_{i}$ who is socially connected to his first choice woman $w_{j}$ then any man who is less preferable than $m_{i}$ in $w_{j}$ preference list, cannot match with $w_{j}$ in any socially stable matching. If it happens, $\left(m_{i}, w_{j}\right)$ will be blocking pair for resultant socially stable matching $M$.

In phase 2, first we build a graph from the reduced instance from phase 1 and weight each edge $\left(m_{i}, w_{j}\right)$ by $\operatorname{rank}\left(w_{j}, m_{i}\right)$, where $\operatorname{rank}\left(w_{j}, m_{i}\right)$ is rank of man $m_{i}$ in $w_{j}$ 's reduced preference list. Now we construct a minimum weight maximum matching $M_{G}$ in graph. Finally, in phase 3 we settle those pairs which are matched in phase 2 but will be socially blocking pair for output matching $M$.

Lemma 4.1.1. $(2, \infty)$-MAX weakly SMTISS algorithm terminates.

Proof. We start phase 1 by unmarking all men. Now we mark those men who are unmarked and have a non-empty reduced list. When every man becomes either marked or having an empty reduced preference list, phase 1 will terminate. Since a man $m_{i}$ can be marked at most twice 
during phase 1 and total number of men in instance $(I, G)$ is finite, phase 1 will terminate. In phase 2 of algorithm we are finding a minimum weight maximum matching of the reduced instance, therefore phase 2 will also terminate. In phase 3, each iteration improves the choice of a man from his second choice woman to his first choice woman and no man obtains worse woman or becomes unmatched. Since total number of possible improvements for men is finite, therefore the total number of iterations is also finite and hence phase 3 will also terminate.

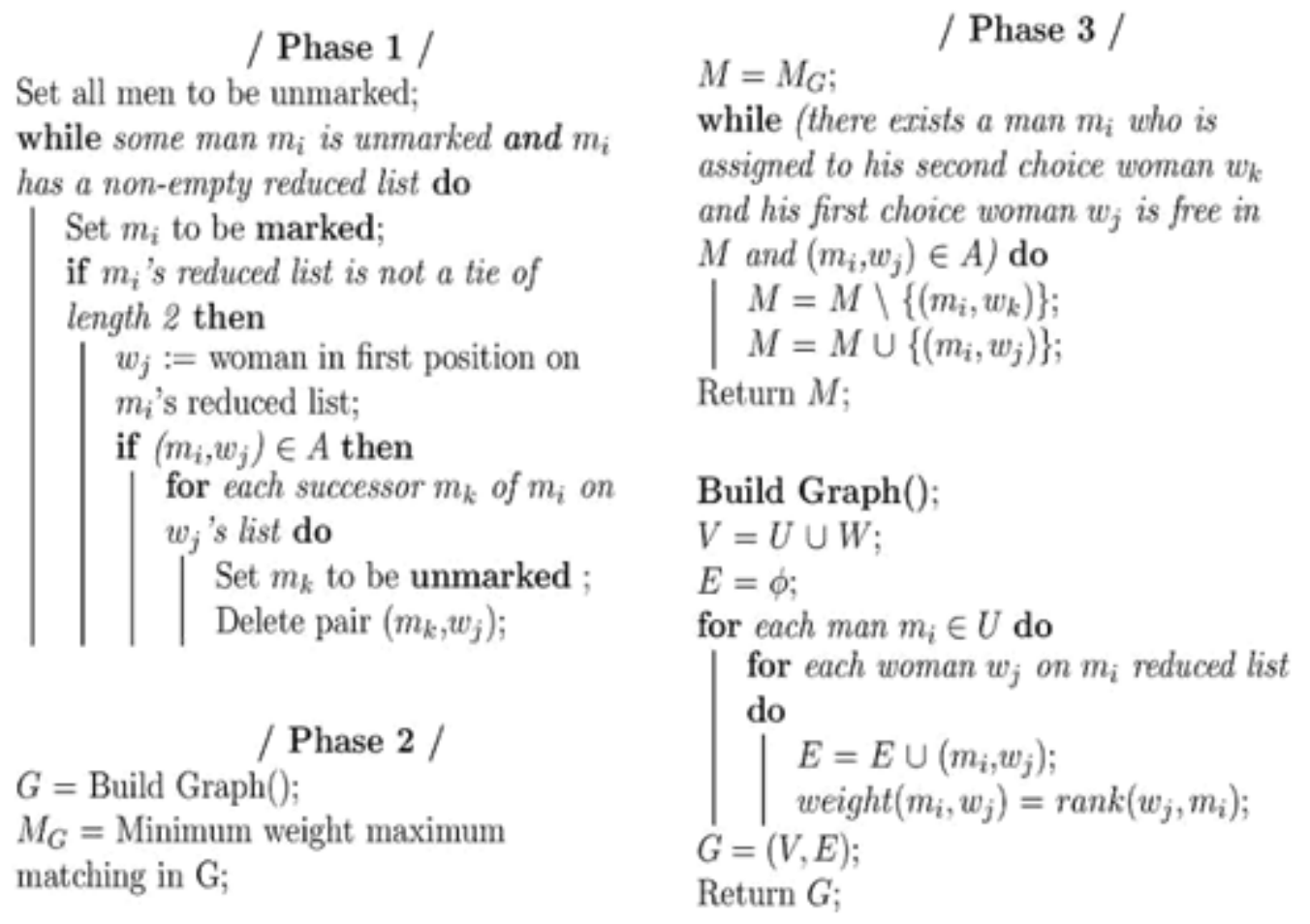

Figure 1. (2, )-MAX Weakly SMTISS Algorithm

Lemma 4.1.2. Phase 1 of $(2, \infty)$-MAX Weakly SMTISS Algorithm never deletes a weakly socially stable pair.

Proof. Suppose $\left(m_{i}, w_{j}\right)$ is a weakly socially stable pair which has been deleted during execution of phase 1 of algorithm 1 such that $\left(m_{i}, w_{j}\right) \in M$, where $M$ is a weakly socially stable matching in $(I, G)$. Suppose this is the first weakly stable pair deleted during phase 1 . This deletion was done because of $w_{j}$ being the first choice of some man $m_{r}$ where $\left(m_{r}, w_{j}\right) \in A, m_{r}$ 's reduced list was not a tie of length 2 and $w_{j}$ prefers $m_{r}$ to $m_{i}$. But in that case pair $\left(m_{r}, w_{j}\right)$ becomes a social blocking pair with respect to matching $M$. This is a contradiction to the fact that $M$ is a weakly socially stable matching.

Lemma 4.1.3. The matching returned by algorithm $(2, \infty)$-MAX weakly SMTISS is weakly socially stable in $(I, G)$.

Proof. Suppose our algorithm outputs the matching $M$ and this matching is not weakly socially stable in $(I, G)$. It means there exist a pair $\left(m_{i}, w_{j}\right)$ which is a socially blocking pair with respect to $M$. We can consider following four cases corresponding to a socially blocking pair. 
Case (i) both $m_{i}$ and $w_{j}$ are unmatched in $M$ :

We know once a man $\mathrm{m}_{\mathrm{i}}$ is matched in $M_{G}$, he will never be unmatched in phase 3 . Either $\mathrm{m}_{\mathrm{i}}$ remains with his partner in $M_{G}$ or he can improve his partner (if possible) during phase 3. Therefore, if a man $m_{i}$ is unmatched in $M$, he was unmatched in $M_{G}$. Woman $w_{j}$ can either be unmatched in $M_{G}$ or during phase 3 . In first case, suppose a woman $w_{j}$ is unmatched in $M_{G}$, then we can increase the size of matching $M_{G}$ by adding the edge $\left(m_{i}, w_{j}\right)$, which contradicts the fact that matching $M_{G}$ is a maximum matching. In second case, suppose a woman $w_{j}$ becomes unmatched during phase 3 , then it means that her partner in $M_{G}$, say $m_{p l}$, had a strict preference list of length 2 and got his first choice woman, say $w_{q 1}$, where $\left(m_{p l}, w_{q 1}\right) \in A$. Now again we have two cases for $w_{q 1}$. In the first case, woman $w_{q l}$ is unmatched in $M_{G}$. This leads to an augmenting path $\left\{\left(m_{i}, w_{j}\right),\left(m_{p l}, w_{j}\right),\left(m_{p l}, w_{q l}\right)\right\}$ in $M_{G}$, where the first and the last edges are not in $M_{G}$. So we can increase the size of the matching $M_{G}$ by one. This is a contradiction to the fact that $M_{G}$ is maximum matching. In second case, suppose $w_{q l}$ becomes unmatched during phase 3, then it means her partner in $M_{G}$, say $m_{p 2}$, had a strict preference list of size 2 and got his first choice woman $w_{q 2}$, where $m_{p 2}$ and $w_{q 2}$ had a social connection in $(I, G)$. Again we can observe an augmenting path $\left\{\left(m_{i}, w_{j}\right),\left(m_{p l}, w_{j}\right),\left(m_{p 1}, w_{q 1}\right)\left(m_{p 2}, w_{q 1}\right),\left(m_{p 2}, w_{q 2}\right)\right\}$ in $\mathbf{M}_{\mathrm{G}}$ which contradicts the fact that $M_{G}$ is maximum matching in $(I, G)$. Similarly, if we keep on doing this operation, number of men is finite and since every man strictly improves his partner in $M_{G}$, there exist a finite number of women who can become unmatched after phase 3. Hence at some time there exists a man $m_{p r}$, who is matched with $w q_{r-1}$ in $\mathrm{M}_{\mathrm{G}}$, and switched to his first choice $w_{q r}$ and $w_{q r}$ is unmatched in $\mathrm{M}_{\mathrm{G}}$. Here we can form an augmenting path $\left\{\left(m_{i}, w_{j}\right),\left(m_{p l}, w_{j}\right),\left(m_{p l}, w_{q 1}\right),\left(m_{p 2}\right.\right.$, $\left.\left.w_{q 1}\right),\left(m_{p 2}, w_{q 2}\right), \ldots,\left(m_{p r}, w_{q r}\right)\right\}$, which leads to a contradiction that $M_{G}$ is a maximum matching.

Case (ii) $m_{i}$ is unmatched in $M$ and $w_{j}$ prefers $m_{i}$ to $M\left(w_{j}\right)$ :

As explained before $\mathrm{m}_{\mathrm{i}}$ is unmatched in $M_{G}$. Woman $w_{j}$ is either matched to $M\left(w_{j}\right)$ in $M_{G}$ or matched to some $m_{p l}$ in $M_{G}$ and after that matched to $M\left(w_{j}\right)$ in phase 3 . In first case, if $w_{j}$ is matched to $M\left(w_{j}\right)$ in $M_{G}$ then it leads to contradiction that $M_{G}$ is a minimum weight maximum matching. We can simply discard the edge $\left(M\left(w_{j}\right), w_{j}\right)$ from $M_{G}$ and add edge $\left(m_{i}, w_{j}\right)$, which reduces the weight of $M_{G}$ without reducing its cardinality. In second case, $w_{j}$ is matched to some $m_{p l}$ in $M_{G}$, where $m_{p l} \neq m_{i} \neq M\left(w_{j}\right)$ and after that matched to $M\left(w_{j}\right)$ in phase 3. Now, after phase $3, w_{j}$ is not matched with $m_{p l}$, which means that $m_{p l}$ got his first choice woman say $w_{q 1}$ in phase 3. Now woman $w_{q 1}$ is either free or matched to some man in $M_{G}$. In both cases, using similar arguments as in Case (i) we can construct an augmenting path and contradict that $M_{G}$ is a maximum matching.

Case (iii) $m_{i}$ is matched to $M\left(m_{i}\right)$ in $M, m_{i}$ prefers $w_{j}$ to $M\left(m_{i}\right)$ and $w_{j}$ is unmatched in $M$ :

We know that man $m_{i}$ has a strict preference list of size 2. It follows that $w_{j}$ is the first woman of $m_{i}$ 's preference list. Since $\left(m_{i}, w_{j}\right)$ is an edge in social graph $G$, this satisfies the loop condition of phase 3 and $\mathrm{m}_{\mathrm{i}}$ will be matched to $w_{j}$ during phase 3 . Therefore this case will never occur after execution of this algorithm.

Case (iv) $m_{i}$ is matched with $w_{k}=M\left(m_{i}\right)$, and $m_{i}$ prefers $w_{j}$ to $w_{k}$ and $w_{j}$ is assigned to $m_{r}=M\left(w_{j}\right)$ and $w_{j}$ prefers $m_{i}$ to $m_{r}$ : 
We know that length of preference list of $m_{i}$ is 2 and $m_{i}$ is in social connection with $w_{j} . m_{i}$ strictly prefers $w_{j}$ to $w_{k}$, which means that $w_{j}$ is first choice of $\mathrm{m}_{\mathrm{i}}$. Woman $w_{j}$ strictly prefers $m_{i}$ to $m_{r}$. Therefore the loop condition of phase 1 will be true and phase 1 will delete the pair $\left(m_{r}, w_{j}\right)$. Hence this case will never occur in our algorithm.

Since by lemma 1 we know that phase 1 of algorithm never deletes a socially stable pair, in phase 2 we constructed a minimum weight maximum matching $M_{G}$ from the reduced preference list by phase 1 using algorithm in [18]. During phase 3 we never decrease the size of $M_{G}$, which follows that resultant matching $M$ after phase 3 is a maximum cardinality matching. Lemma 3 ensures that the matching produced by $(2, \infty)$-MAX weakly SMTISS algorithm is weakly socially stable. It follows that the algorithm produced a maximum weakly socially stable matching in instance ( $I$, $G)$. The running time complexity of the algorithm is dominated by phase 2 which constructs a minimum weight maximum matching in $G^{\prime}\left(V, E^{\prime}\right)$ in time $O\left(\sqrt{ }|V|\left|E^{\prime}\right| \log |V|\right)$ [18]. Suppose $|V|=n$ $=n_{1}+n_{2}$ is total number of men and women, then the cardinality of set of acceptable pairs is at most $2 n_{1}=\mathrm{O}(n)$. It follows that the time complexity of $(2, \infty)$-MAX weakly SMTISS algorithm is $O\left(n^{3 / 2} \log n\right)$.

Theorem 4.1. For a given instance (I, G) of $(2, \infty)$-MAX Weakly SMTISS, Algorithm $(2, \infty)$ MAX weakly SMTISS produces a maximum size weakly socially stable matching in $O\left(n^{3 / 2} \log n\right)$ time, where $n$ is the total number of men and women in $I$.

\section{CONCLUSION AND FUTURE WORK}

In this paper we have presented an algorithm for an instance of stable marriage problem with ties and incomplete bounded length preference list, where each man can give at most 2 women in his preference list (with or without ties) and each woman can give unbounded length preference list (with or without ties). Length of ties in women preference list could be 2 or more. We have found that this instance can be solved in polynomial time. These instances are very common in real world scenario like NRMP and Scottish medical matching scheme where medical students can give small size preference list. It would be interesting to study about maximum size strongly stable matching and super stable matching in the scenario of social stability. We leave this as an open problem.

\section{ACKNOWLEDGEMENTS}

The authors are grateful to Dr. Meghana Nasre for useful discussion on the problem and anonymous reviewers for their useful comments which improved the quality and presentation considerably.

\section{REFERENCES}

[1] Gale, D., Shapley, L.S.: College admissions and the stability of marriage. The American Mathematical Monthly 69 (1962) 9-15

[2] Gusfield, D., Irving, R.W.: The Stable Marriage Problem: Structure and Algorithms. MIT Press, Cambridge, MA, USA (1989) 
[3] Irving, R.W.: Stable marriage and indifference. In: Selected Papers of the Conference on Combinatorial Optimization. CO89, Amsterdam, The Netherlands, The Netherlands, North-Holland Publishing Co. (1994) 261-272

[4] Kavitha, T., Mehlhorn, K., Michail, D., Paluch, K.E.: Strongly stable matchings in time o(nm) and extension to the hospitals-residents problem. ACM Trans. Algorithms 3 (2007)

[5] Iwama, K., Manlove, D., Miyazaki, S., Morita, Y.: Stable marriage with incomplete lists and ties. In: In Proceedings of ICALP 99: the 26th International Colloquium on Automata, Languages and Programming, Springer-Verlag (1999) 443-452

[6] Manlove, D.F., Irving, R.W., Iwama, K., Miyazaki, S., Morita, Y.: Hard variants of stable marriage. Theoretical Computer Science 276 (2002) 261-279

[7] Halldrsson, M.M., Irving, R.W., Iwama, K., Manlove, D.F., Miyazaki, S., Morita, Y., Scott, S.: Approximability results for stable marriage problems with ties. Theoretical Computer Science 306 (2003) 431-447

[8] Halldrsson, M.M., Iwama, K., Miyazaki, S., Morita, Y.: Inapproximability results on stable marriage problems. In Rajsbaum, S., ed.: LATIN. Volume 2286 of Lecture Notes in Computer Science., Springer (2002) 554-568

[9] Halldorsson, M.M., Iwama, K., Miyazaki, S., Yanagisawa, H.: Improved approximation results for the stable marriage problem. ACM Trans. Algorithms 3 (2007)

[10] Halldrsson, M.M., Iwama, K., Miyazaki, S., Yanagisawa, H.: Randomized approximation of the stable marriage problem. Theoretical Computer Science 325 (2004) 439-465 Selected Papers from $\{$ COCOON\} 2003.

[11] Iwama, K., Miyazaki, S., Okamoto, K.: A (2-c( $\log n / n))$-approximation algorithm for the stable marriage problem. In Hagerup, T., Katajainen, J., eds.: SWAT. Volume 3111 of Lecture Notes in Computer Science., Springer (2004) 349-361

[12] Iwama, K., Miyazaki, S., Yamauchi, N.: A (2-c(1/sqrt(n)))-approximation algorithm for the stable marriage problem. Algorithmica 51 (2008) 342-356

[13] Gale, D., Sotomayor, M.: Some remarks on the stable matching problem. Discrete Applied Mathematics 1 (1985) 223-232

[14] Irving, R.W.: Matching medical students to pairs of hospitals: A new variation on a well-known theme. In Bilardi, G., Italiano, G.F., Pietracaprina, A., Pucci, G., eds.: ESA. Volume 1461 of Lecture Notes in Computer Science., Springer (1998) 381-392

[15] Garey, M., Johnson, D., Stockmeyer, L.: Some simplified np-complete graph problems. Theoretical Computer Science 1 (1976) 237-267

[16] Irving, R.W., Manlove, D.F.: Stable marriage with ties and bounded length preference lists. In: In Proc. ACID, Volume 7 of Texts in Algorithmics, 95106, (College Publications)

[17] Askalidis, G., Immorlica, N., Kwanashie, A., Manlove, D., Pountourakis, E.: Socially stable matchings in the hospitals/residents problem. In Dehne, F., Solis-Oba, R., Sack, J.R., eds.: Algorithms and Data Structures. Volume 8037 of Lecture Notes in Computer Science. Springer Berlin Heidelberg (2013) 85-96 
[18] Gabow, H.N., Tarjan, R.E.: Faster scaling algorithms for network problems. SIAM J. COMPUT 18 (1989) 1013-1036

\section{AUTHORS}

Ashish Shrivastava is a M.S.(By Research) student in Computer Science and Engineering department in Indian Institute of Technology, Madras Chennai. His area of research is Theoretical Computer Science.

C.Pandu Rangan is a Professor in Computer Science and Engineering department in Indian Institute of Technology, Madras Chennai. His area of research is Theoretical Computer Science, Cryptography.

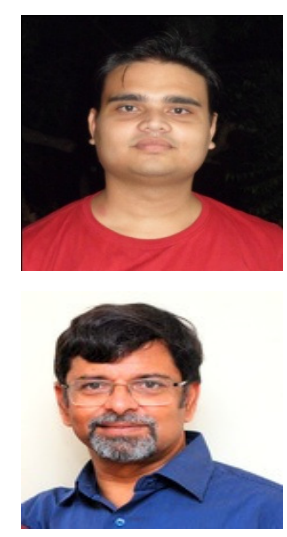

